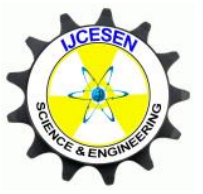

Copyright (C) IJCESEN
International Journal of Computational and

Experimental $\boldsymbol{S}$ cience and $\boldsymbol{E N g i n e e r i n g}$

(IJCESEN)

Vol. 5-No.2 (2019) pp. 72-75

http://dergipark.org.tr/ijcesen

Research Article

\title{
Investigation of Various Infectious Diseases in Turkey by Mathematical Models SI and SIS
}

\author{
Arzu ÇíLLi ${ }^{1} *$, Kıvanç ERGEN ${ }^{2}$ \\ ${ }^{1}$ Yildiz Technical University, Science and Arts Faculty, Physics Department, 34210, İstanbul-TÜRKIYE \\ ${ }^{2}$ İzmir Democracy University, Medical Faculty, Biophysics Department, İzmir-TÜRKIYYE \\ * Corresponding Author: acilli@yildiz.edu.tr \\ ORCID: 0000-0002-0596-3637
}

\begin{abstract}
Article Info:
Received : 20 March 2019

Accepted : 15 July 2019

\section{$\underline{\text { Keywords }}$}

Infectious

Diseases

Mathematical Model

Susceptible

Prediction
\end{abstract}

DOI: $10.22399 /$ ijcesen.542383

\begin{abstract}
:
Mathematical models can give us information on number of cases, deaths caused by infectious diseases. In this study, we aimed to predict the effects of CrimeanCongo Hemorrhagic Fever (CCHF), tuberculosis (TB), measles for Turkey and the efficiency of SI and SIS mathematical models were defined in the prediction of the number of infected people for these diseases. In CCHF, predictions for 2014 gave $0 \%$ error in both models. As for TB, SIS model predicted the exact number and SI model predicted a few more cases than that of SIS model. Again SI and SIS models gave the exact values for measles. According to these predictions it seems that $\mathrm{CCHF}$ and $\mathrm{TB}$ cases will continue to increase slightly while measles cases will approach zero.

These models can predict exact numbers for each year, in a long term and in normal conditions (unless there are external parameters such as natural disaster, war, emigration and terrorism ) they can predict the trend for the diseases and can tell when to disappear. Therefore, updating data are of importance to achieve the powerful prediction.
\end{abstract}

\section{Introduction}

Epidemic diseases are the presence of diseases or infectious agents within a given geographic area or population group. It may also refer to the usual prevalence of a given disease with such area or group. Epidemic diseases have been an important problem for mankind. Among them are lyme disease (1975) [1], legionella (1976) [2], AIDS (Acquired Immune Deficiency Syndrome) (1981) [3], hepatitis C (1989) [4], Severe Acute Respiratory Syndrome (SARS) [6] appearing in recent years.

Fighting with epidemic diseases primarily begins prior to epidemia. Taking appropriate precautions and foreseeing the impact of the diseases are of importance to reduce/minimize the negative social and economic effects. Not only therapy and protection methods, but also prediction of the number of cases, deaths and spread rate are very useful.
At this point, mathematical models can give us information on number of case, death caused by these diseases. Therefore we can predict the case/death of a specific disease by using past data of that specific area/country, although there could be some deviations arise from great changeless in community such as emigration, etc.

In this project we aimed to predict the effects of Crimean-Congo Hemorrhagic Fever (CCHF), Tuberculosis and Measles for Turkey by using 2013 and 2014 data. Predictions in terms of the number of infected people were made for 2014 and 2015 by using mathematical modelling SI (SusceptibleInfected) and SIS (Susceptible-InfectedSusceptible). We aimed to find the best model for a specific disease for a specific country in the light of the results. Predictive power of the models in general was discussed for its reliability and robustness.

\section{Epidemiological Models: SI and SIS}


One of the mathematical epidemiological models that is used for theorizing about and emulating epidemics is the SIS model $[7,8,9]$. This model is implemented by an iterative calculation. In this model, which is effective for epidemic diseases, the simplest version without demographic factors is defined as follows. It is assumed that there are no deaths, no births in the population, no migration into and out of the population and infected people are not re-infected after they have recovered.

\subsection{Formulation of SI and SIS Models}

In SI model, over the course of the epidemic almost everyone eventually becomes infected. In the SIS model, each individual belongs to either the set of susceptibles (S) or the set of infected people (I). Thus, the model can be shown as $\mathbf{S} \rightarrow \mathbf{I} \rightarrow \mathbf{S}$, that is, a person is either susceptible or infected. After being infected, he/she can be suscetible again (i.e.getting recovered). When a susceptible individual and an infected individual interact (by some manner), the former may be infected at a rate of infection, denoted by a constant $\beta$. In this way, susceptibility is the same for each individual. By accepting these rules following equations are obtained [8]. Here we consider the SI and SIS models where the total population;

$$
S(t)+I(t)=N
$$

is a constant and assumed that it does not change with time.

For SI model;

$$
\begin{aligned}
& \frac{d S}{d t}=\left(-\frac{\beta}{N}\right) S(t) I(t) \\
& \frac{d I}{d t}=\left(\frac{\beta}{N}\right) S(t) I(t)
\end{aligned}
$$

For SIS model;

$$
\begin{aligned}
& \frac{d S}{d t}=\left(-\frac{\beta}{N}\right) S(t) I(t)+\gamma I(t) \\
& \frac{d I}{d t}=\left(\frac{\beta}{N}\right) S(t) I(t)-\gamma I(t)
\end{aligned}
$$

By using Eq.(4) and Eq.(5), firstly $\gamma$ is found as follows;

$$
\gamma=\frac{\left(\frac{d R}{d t}\right)}{I(t)}
$$

And then $\beta$ is calculated from Eq.(5).

SIS turns into SIR model (Susceptible-InfectedRecovered) if (R) Recovery is calculated [12]. In SIS, R is excluded. For Eq's.(2-6), we accept the number of people leaving the susceptible groups is equal to incoming people to infected group (I). In the meantime infected people can recover and this average recovering ratio is symbolized by $\gamma$ and $1 / \gamma$ denotes the average infectious period. When time $t$ approaches infinity, $\mathrm{S}$ and $\mathrm{R}$ approach ' $O$ ' and ' $N$ ' respectively. For solving the differential Eq.(2) and Eq.(3), the Euler Method is used. A susceptible individual may get in contact with an infected individual with probability $\beta$ within a short time interval $\Delta t$.

With high probability $(1-\beta \Delta t)$, the susceptible individual stays in the susceptible state throughout the interval $\Delta t$ and this interval $\Delta t$ can be identified with a discrete simulation time step.

\subsection{Euler Method}

Euler equations are given by discretization of the difference Eq's (2-5) and Eq's (4-5); for SI and SIS respectively;

For SI Model;

$$
\begin{aligned}
S_{n+1} & =S_{n}-\left(\frac{\beta}{N}\right) S_{n} I_{n} \\
I_{n+1} & =I_{n}\left[1+\left(\frac{\beta}{N}\right) S_{n} \Delta t\right]
\end{aligned}
$$

For SIS Model;

$$
S_{n+1}=S_{n}-\left(\frac{\beta}{N}\right) S_{n} I_{n}+\gamma_{n}
$$

$$
I_{n+1}=I_{n}\left[1+\left(\frac{\beta}{N}\right) S_{n} \Delta t\right]-\gamma_{n}
$$

Population in the next step can easily be determined in very short time intervals. To solve these equations, firstly the ratios $\beta$ and $\gamma$ (used in SIS model) had to be known. An approximate prediction on infected and healthy people can be made by knowing these $\beta$ and $\gamma$ ratios for a fixed number of populations in defined time intervals [8].

\section{Results}

It is assumed that population is fixed in the SI and SIS models. $\beta$ values were calculated for each disease using the related values of two previous 
consecutive years. $\beta$ was used for the following years as can be seen from the Tables, thus predicted results were given. As mentioned before, this weak model is unable to estimate the number of dead people so we cannot give the number of deaths. In the following calculations, population of Turkey was taken as 78.741.053.

\subsection{CCHF(Crimean-Congo Hemorrhagic Fever) Cases and Predictions in Turkey}

Crimean-Congo hemorrhagic fever (CCHF) is a widespread disease caused by a virus. The CCHF virus causes severe viral hemorrhagic fever outbreaks, with a case fatality rate of $10-40 \%$. Since 2000, CCHF caused many cases and deaths in Turkey.

Table 1. Number of CCHF (Crimean-Congo Hemorrhagic Fever) Cases and Predictions in Turkey

\begin{tabular}{|c|c|c|c|c|c|c|}
\hline Years & \multirow{2}{*}{ Cases } & \multicolumn{2}{|c|}{ SI } & \multicolumn{3}{|c|}{ SIS } \\
\cline { 3 - 7 } & & $\boldsymbol{\beta}$ & Prediction & $\boldsymbol{\gamma}$ & $\boldsymbol{\beta}$ & Prediction \\
\hline 2013 & 910 & ---- & ---- & ---- & ---- & ---- \\
\hline 2014 & 967 & 0,063 & 967 & 0,959 & 1,022 & 967 \\
\hline 2015 & ---- & 0,063 & 1028 & 0,959 & 1,022 & 1028 \\
\hline 2016 & ---- & 0,063 & 1092 & 0,959 & 1,022 & 1092 \\
\hline
\end{tabular}

We have tried to predict 2015 and 2016 by using SI and SIS models. Predictions for 2014 gave 0\% error in both models. According to these predictions CCHF will continue to increase slightly.

\subsection{Tuberculosis Cases and Predictions in Turkey}

Tuberculosis (TB) is an infectious disease caused by the bacterium Mycobacterium tuberculosis (MTB). Tuberculosis generally affects the lungs, but can also affect other parts of the body. Tuberculosis is still the most mortal epidemic disease each year in Turkey. Although data give the information that mortality is getting lower, today many tuberculosis cases result in death.

Table 2. Number of Tuberculosis Cases and Predictions in Turkey

\begin{tabular}{|c|c|c|c|c|c|c|}
\hline Years & \multirow{2}{*}{ Cases } & \multicolumn{2}{|c|}{ SI } & \multicolumn{3}{|c|}{ SIS } \\
\cline { 3 - 7 } & & $\boldsymbol{\beta}$ & Prediction & $\boldsymbol{\gamma}$ & $\boldsymbol{\beta}$ & Prediction \\
\hline 2013 & 13409 & ---- & ---- & ---- & ---- & ---- \\
\hline 2014 & 13378 & $-0,002$ & 13382 & 0,976 & 0,974 & 13378 \\
\hline 2015 & ---- & $-0,002$ & 13355 & 0,976 & 0,974 & 13347 \\
\hline 2016 & ---- & $-0,002$ & 13328 & 0,976 & 0,974 & 13316 \\
\hline
\end{tabular}

High mortality can be explained mainly by being the most tuberculosis patients are also HIV (+). SIS model predicted the exact number. SI model predicted a few more cases than that of SIS model. These predictions show that the tuberculosis cases will continue in a slightly increasing manner.

\subsection{Measles Cases and Predictions in Turkey}

Measles is a highly contagious infection caused by the virus. Measles is a spreading disease especially in small age children. We predicted the cases for 2014 and 2015. Although the cases were dropped to 3-7 cases in 2000's, it increased again after major migrations from 2010 onwards. As of 2013, it is seen under control and decreased number in transporter migrants. So that, Measles has proved that SI and SIS models can give proper results.

Table 3. Number of Measles Cases and Predictions in

\begin{tabular}{|c|c|c|c|c|c|c|}
\hline \multirow{2}{*}{ Years } & \multirow{2}{*}{ Cases } & \multicolumn{2}{|c|}{ SI } & \multicolumn{3}{c|}{ SIS } \\
\cline { 3 - 7 } & & $\beta$ & Prediction & $\gamma$ & $\beta$ & Prediction \\
\hline 2013 & 7465 & ---- & ---- & --- & ---- & ---- \\
\hline 2014 & 565 & -0924 & 565 & 0,999 & 0,753 & 565 \\
\hline 2015 & ---- & -0924 & 43 & 0,999 & 0,753 & 43 \\
\hline 2016 & ---- & -0924 & 3 & 0,999 & 0,753 & 3 \\
\hline
\end{tabular}

Here as in the case of CCHF, SI and SIS models gave the exact values. Providing the exact numbers can be explained that mortality and cases are not high and do not show big changeness in consecutive years. According to these values, Measles will be under control extensively in 2015 and 2016. Number of cases limit to zero, that is to say a few or none.

\section{Conclusion}

In this study, three epidemic diseases were investigated using the epidemiological models SI and SIS. We predicted the number of infected people to present day for these diseases results showed that SI and SIS models give similar or same values where cases and deaths are low and yearly changeness is not big. Therefore both models were found to confirm the reliability and robustness. They can predict the number of infected individuals well. This is sensitive to fluctuations in real data and able to predict the trend of the exact data and gives good results in a short time. In general these models in specified regions/countries give 
predictions close to real values unless there are external parameters such as natural disaster, war, emigration and terrorism. Even though models do not predict exact numbers for each year, in a long term and in a normal conditions they can predict the trend for the diseases and can tell when to disappear. Therefore, updating data are of importance to achieve this.

We have also implemented these models for epidemic diseases in some countries around the world. [13]

\section{References}

[1] Steere AC (1989) Lyme disease. N Engl J Med 321: 586-596.

[2]Hilbi H, Jarraud S, Hartland E, Buchrieser C (2010) Update on Legionnaires' disease: pathogenesis, epidemiology, detection and control. Mol Microbiol 76: 1-11.

[3]Taff ML, Siegal FP, Geller SA. Outbreak of an acquired immunodeficiency syndrome associated with opportunistic infections and Kaposi's sarcoma in male homosexuals. Am. J.Forensic Med.Pathol. 1982 Sep.3(3):259-64.

[4]Zuckerman AJ. The Elusive Hepatitis C Virus. BMJ. 1989 Oct 7;299(6704):871-3.

[5]Drosten C, Günther S, Preiser W, et all. Identification of a Novel Coronavirus in Patients with Severe Acute Respiratory Syndrome, N Engl J Med. 2003 May 15;348(20):1967-76. Epub 2003 Apr 10.

[6]Masuda N, Holme P. Predicting and controlling infectious disease epidemics using temporal networks. F1000Prime Rep. 2013;5:6. doi: 10.12703/P5-6. Epub 2013 Mar 4.

[7]Lih-Ing W. Roeger. Dynamically Consistent DiscreteTime SI and SIS Epidemic Models, Discrete and Continuous Dynamical systems, supplement,653-662, 2013.

[8]Keeling MJ, Rohani P: Modeling Infectious Diseases in Humans and Animals. Princeton: Princeton University Press; 2007.

[9]www.thsk.gov.tr/component/k2/zoonotik-ve-vektorelhastaliklar-daire-baskanligi-istatiksel- verileri.html

[10]www.thsk.gov.tr/component/k2/tuberkuloz-dairebaskanligi-istatiksel-verileri.html

[11] www.thsk.gov.tr/component/k2/asi-ile-onlenebilirhastaliklar-daire-baskanligi- istatikselverileri.html

[12] Ergen K., Çilli A. and Yahnioğlu N., Predicting Epidemic Diseases using Mathematical Modelling of SIR, DOI:10.12693/APhysPolA.128.B-273.

[13]Çilli A., Ergen K., Akat E., "Some Mathematical Models and Applications Used in Epidemics", SIGMA Journal of Engineering and Natural Sciences", vol.37, no.1, pp.17-23, 2019. 